\title{
NECROSIS DE PIEL DE PENE COMO COMPLICACIÓN AL USO DE COLECTOR URINARIO
}

\author{
J.L. PALMERO MARTÍ, M.A. BONILLO GARCÍA, J.J. PACHECO BRU, \\ J.M. ALAPONT ALACREU, L. GARCÍA REBOLL, J.F. JIMÉNEZ CRUZ.
}

Servicio de Urología. Hospital Universitario La Fe. Valencia.

Actas Urol Esp. 27 (2): 155-158, 2003

\section{RESUMEN}

“NECROSIS DE PIEL DE PENE COMO COMPLICACIÓN AL USO DE COLECTOR URINARIO"

Presentamos un caso de necrosis de la piel del pene en un paciente con incontinencia por vejiga neurógena secundaria a lesión medular traumática. Ésta se produjo como consecuencia de un exceso de presión provocado por un colector urinario. Presentamos el caso considerando que este tipo de complicaciones son poco frecuentes, siendo escasas las referencias en la literatura sin embargo dada la elevada frecuencia de pacientes incontinentes que emplean este tipo de dispositivos, es necesario conocer las posibles consecuencias derivadas de un uso incorrecto de los mismos.

PALABRAS CLAVE: Necrosis piel de pene. Colector de orina. Vejiga neurógena por lesión medular.

\begin{abstract}
"SKIN PENILE NECROSIS AS A COMPLICATION FROM URINARY CONDOM CATHETER USES"

We illustrate a case of penile skin necrosis in a patient suffering from urinary incontinence caused by a secondary neurogenic bladder that, in turn, results from a spinal cord injury. The skin necrosis developed out of continuous pressure from the condom catheter. We report the case bearing into consideration that these complications are extremely rare and that references in the literature to the topic are likewise scarce. However, due to the high frequency of patients suffering from incontinence who use these devices, we believe it necessary to account for the possible consequences which might derive from an incorrect use of them.
\end{abstract}

KEY WORDS: Penile skin necrosis. Condom catéter. Neurogenic bladder in spinal cord injury.

$\mathrm{L}$ a vejiga neurógena se define como las alteraciones del funcionamiento vésico-uretral secundarias a lesión neurológica. La causa más frecuente es la lesión medular, en especial de tipo traumático, que ocurre más frecuentemente en adolescentes y adultos jóvenes. La causa principal de este tipo de lesiones sigue siendo los accidentes de tráfico. El empleo de colectores urinarios para evitar los efectos secundarios de los escapes de orina y mejorar la higiene perso- nal del paciente, se considera como una opción terapéutica ampliamente aceptada, en el tratamiento de la vejiga neurógena que genera incontinencia, en aquellos casos en los que no es posible o fracasa el tratamiento quirúrgico, o bien, el empleo de fármacos no mejora la sintomatología o esta contraindicado. A pesar de ello, son pocas las referencias en la literatura, acerca de las complicaciones que se pueden derivar del uso de los mismos. 


\section{CASO CLÍNICO}

Paciente varón de 22 años, con vejiga neurógena secundaria a lesión medular de 12 meses de evolución, tras accidente de circulación, consecuencia del cual se produjo fractura-luxación C5C6 con lesión medular incompleta y fractura-luxación T9-T10 con lesión medular completa nivel T7. Tras el tratamiento quirúrgico de las fracturas, con fijación de las mismas, y una vez restablecido de la fase de shock medular, se practicó un estudio neurourológico al paciente apreciándose: aumento de la actividad del detrusor con aparición de contracción no inhibida del detrusor en fase precoz del llenado (hiperreflexia) que generaba incontinencia, así como, disfunción vesical de vaciado por disinergia vésico-esfinteriana. Entre otros antecedentes personales señalar una politoxicomanía y trastorno de personalidad disocial.

Este paciente inició tratamiento combinado con alfabloqueante y anticolinérgico, no acudiendo a los controles programados para su seguimiento evolutivo. A los doce meses de la lesión medular, acude al servicio de urgencias de nuestro hospital por tumefacción progresiva del pene de 48 horas de evolución. Refiere que lleva usando colectores de orina varios meses, ya que le resulta más cómodo para el control de su incontinencia (había abandonado el tratamiento farmacológico por propia voluntad). Dos días antes de su ingreso, había recambiado el colector urinario, produciéndose una edematización progresiva del pene sin ninguna otra sintomatología asociada.

En el examen físico el paciente se encontraba febril y presentaba una úlcera exudativa en el tercio proximal del cuerpo del pene, que abarcaba toda la circunferencia del mismo, consecuencia de una excesiva presión que el colector provocaba sobre la zona ulcerada. Distal a la lesión ulcerosa, se apreciaba intensa edematización del pene con áreas de equimosis y zonas de desprendimiento de la epidermis. En el borde del prepucio existía una mínima zona de necrosis que en pocas horas fue aumentando su tamaño de manera progresiva a pesar de la retirada inmediata del colector y de la instauración de antibioticoterapia de amplio espectro. Era imposible visualizar el glande debido al intenso edema. (Figs. 1a y lb).

En la exploración complementaria que se realizo destaca la presencia de importante leucoci-
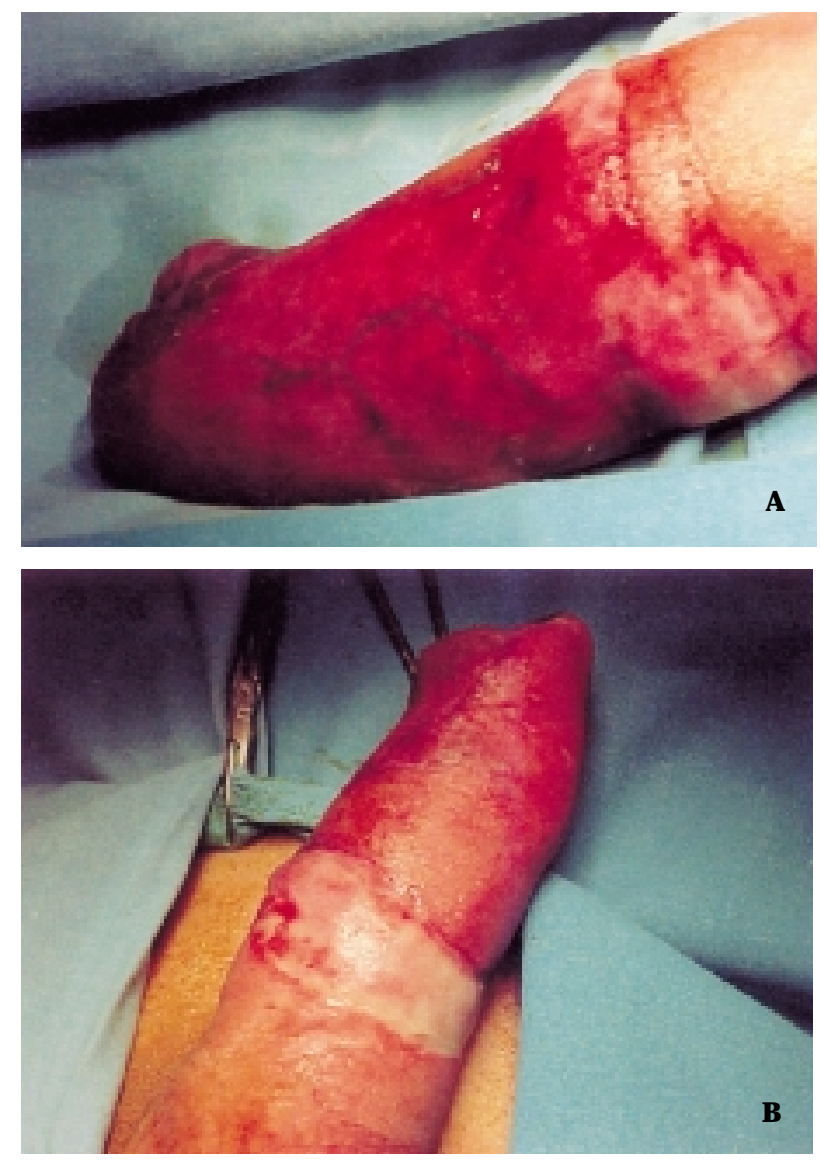

FIGURA 1. 1A: Zona de piel comprometida por la isquemia en toda su extensión, apreciándose la ulcera exudativa. 1B: Detalle de la ulcera provocada por el adhesivo de fijación.

tosis con desviación a la izquierda de la fórmula leucocitaria (21400 leucocitos con $89 \%$ de neutrófilos).

Ante el empeoramiento progresivo de la lesión, con extensión del área de necrosis por la isquemia a que habían estado sometidos los tejidos, y la afectación clínica (fiebre) y analítica del paciente, se decide proceder a intervención quirúrgica urgente. Durante la misma se practica desbridamiento de tejidos necróticos hasta alcanzar tejidos vascularizados, que se extiende a toda la piel y tejido celular subcutáneo desde la zona ulcerada hasta el borde del prepucio (Fig. 2).

Se dejó sonda vesical y se protegió la zona con Tulgrasum antibiótico. La evolución fue satisfactoria, realizándose curas locales diarias y cuidados de sonda vesical, apareciendo de manera progresiva buen tejido de granulación sin progresión de la necrosis ni formación de esfácelos (Fig. 3). 


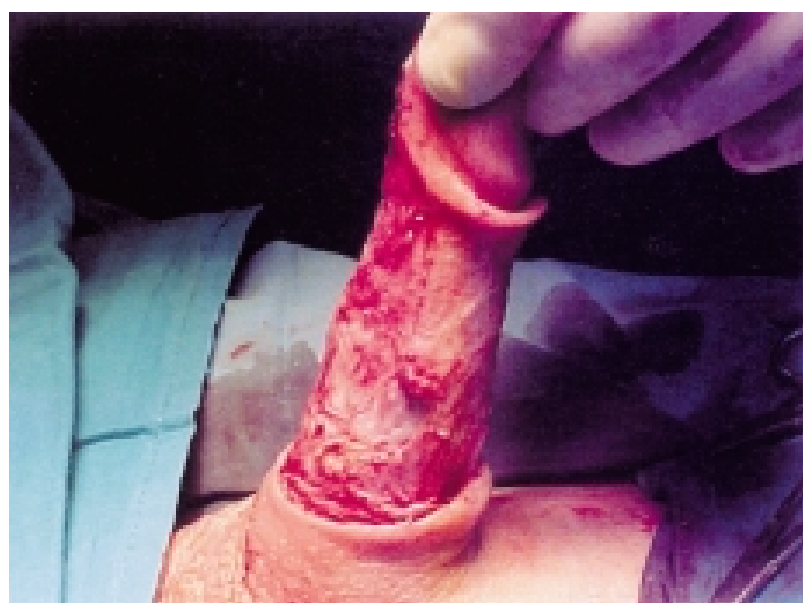

Figura 2. Imagen tras el desbridamiento. Se aprecia la buena vascularización tisular tras el acto quirúrgico.

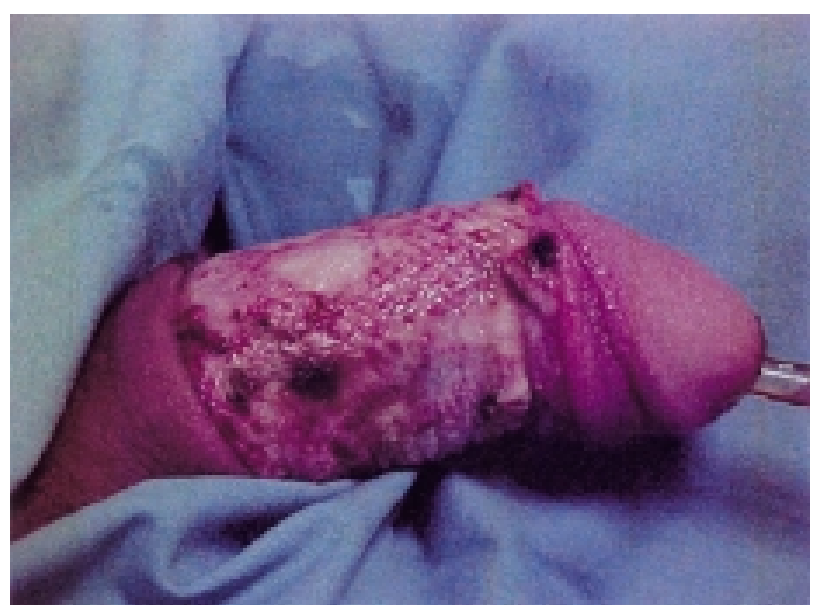

Figura 3. Tejido de granulación al $5^{\circ}$ dia del postoperatorio.

A los diez días del desbridamiento, estando la zona recubierta de tejido de granulación con una buena vascularización del mismo, se procedió a cubrir la zona con un injerto de piel libre, obtenido de la cara externa del muslo derecho, que se suturó con puntos sueltos de material reabsorbible, dejado en toda su extensión pequeñas incisiones de descarga (Fig. 4).

En las dos semanas posteriores se realizaron curas periódicas de la zona, con buen prendimiento del injerto. $\mathrm{El}$ paciente fue dado de alta a los 25 días del ingreso.

\section{DISCUSIÓN}

La causa más frecuente de vejiga neurógena es la lesión medular, siendo las lesiones de tipo traumático las más frecuentes ${ }^{1,5}$. La incidencia anual

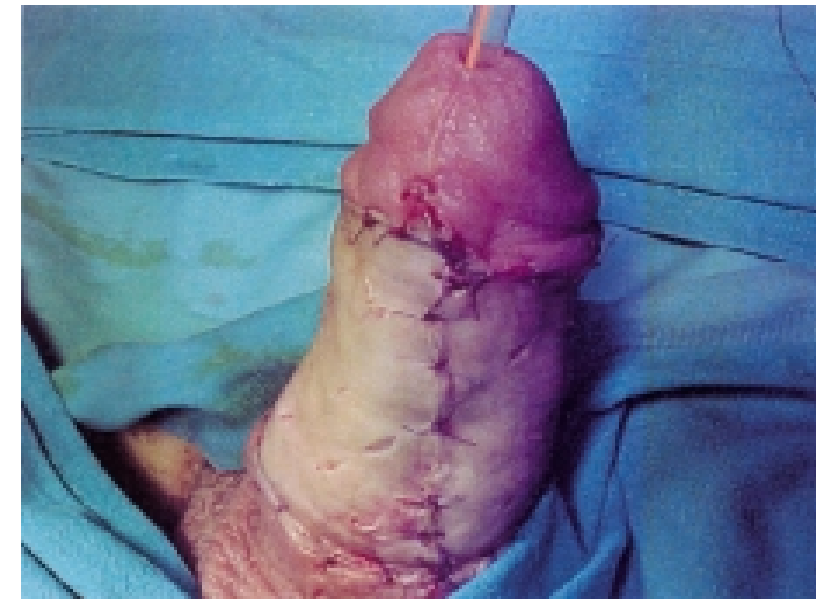

Figura 4. Injerto de piel libre obtenida del muslo, con incisiones de descarga sobre el mismo.

de lesión medular es de treinta y cuatro casos por millón de habitantes en Estados Unidos. En España se producen en torno a doscientos nuevos casos de lesión medular al año, siendo la proporción hombre/mujer de 4 a 1, ocurriendo más frecuentemente en adolescentes y adultos jóvenes. En España, la causa principal de lesión medular sigue siendo los accidentes de tráfico, produciéndose en estos impactos violentos, luxación y/o fractura de cualquier vértebra (como en el caso que acabamos de presentar) que conllevan una sección de la médula espinal con la consiguiente pérdida de todas las funciones motoras, sensitivas, de tipo autónomo y esfinterianas por debajo del nivel de lesión ${ }^{5}$.

La lesión de estructuras nerviosas que intervienen en el control de la micción origina diversos tipos de vejiga neurógena, siendo en este caso un cuadro de disinergia vésico-esfinteriana con hiperreflexia que causaba clínica mixta con predominio de la incontinencia, motivo por el cual el paciente comenzó con el uso de colectores urinarios.

El empleo de este tipo de dispositivos es fácil y se usa frecuentemente en pacientes que padecen este tipo de lesiones y que sufren incontinencia importante. Sin embargo, son escasas las referencias en la literatura en cuanto a complicaciones surgidas por uso de los mismos ${ }^{1}$. En la literatura revisada se comunica la aparición de diversos tipos de lesiones, consecuencia de un uso indebido de colectores, siendo provocadas la mayoría por un efecto constrictor prolongado de la 
banda adhesiva de estos dispositivos para una mejor fijación ${ }^{1-4}$. La mayoría de estas complicaciones aparecen fuera del ámbito sanitario donde el uso de este tipo de dispositivos queda en manos del propio paciente y de personal no sanitario ${ }^{1}$. Dicho exceso de presión, originaría una isquemia progresiva con la consiguiente desvitalización tisular, que inicialmente se manifestaría con la aparición de una zona ulcerada en la piel sobre la cual se está ejerciendo el exceso de presión ${ }^{1}$. Por otro lado, la analgesia y anestesia por debajo del nivel de lesión que padecen estos enfermos, favorece la producción de este tipo de lesiones, al carecer de sintomatología que alerte de una aplicación no adecuada de este tipo de sistemas ${ }^{1-4}$. Este cúmulo de circunstancias se presentan en el caso que aportamos, donde un paciente con una lesión medular postraumática de tipo completo con un nivel dorsal que genera incontinencia y que le lleva al empleo de colector urinario, sufre los efectos de una excesiva presión de la banda adhesiva de fijación del mismo, produciéndose un rodete de isquemia en la zona de presión con aparición de una zona anular ulcerada sobre la piel del pene donde estaba aplicada, y la consiguiente desvitalización de los tejidos a partir del área de isquemia. En este caso se pone de manifiesto que la falta de sensibilidad táctil y nociceptiva, consecuencia de la lesión medular, favoreció el desarrollo de la necrosis de la piel del pene, limitándose el daño a la misma, ya que, el tiempo de evolución no fue excesivamente prolongado.

En la prevención de este tipo de complicaciones, disponemos de escaso margen para actuar sobre lesión medular establecida, mientras que si debemos hacerlo en el manejo de colectores, donde es importante la educación o instrucción acerca del empleo de este tipo de dispositivos ${ }^{1,2,4}$, tanto al propio paciente como al personal que se encarga de su atención. La movilización del dispositivo al menos una vez al día y la aplicación de la banda de fijación en zonas de piel diferentes con el fin de evitar los efectos de la misma sobre una única área de la piel de pene ${ }^{1}$. Además es importante incidir en una adecuada higiene de la región genital, ya que este tipo de dispositivos se han relacionado con una mayor incidencia de infecciones urinarias y juegan un papel importante como reservorio de patógenos en infecciones crónicas del tracto urinario ${ }^{1}$.

\section{CONCLUSIONES}

El empleo de colectores es frecuente para el tratamiento de incontinencia urinaria, siendo frecuente su uso en pacientes con vejiga neurógena secundaria a lesión medular. Consideramos que dadas las especiales características de este tipo de pacientes, tanto éstos, como sus familiares y personal encargado de su cuidado, deben ser instruidos en la correcta utilización de los colectores urinarios, que si bien, son seguros en su uso, ya que son pocos los casos de complicaciones derivadas de su empleo, pueden producir complicaciones por un uso inapropiado de los mismos que deben conocerse y ser tenidas en consideración.

\section{REFERENCIAS}

1. JAYACHANDRAN S, UNNI M. M, MOOPPAN MD.: Complications from external (condom) urinary drainage devices. Urology 1985; 25: 31-34.

2. MELEKOS MD, ASBACH HW.: Complications from urinary condom catheters. Urology 1986; 27: 88.

3. STEINHARDT G, Mc ROBERTS JW.: Total distal penile necrosis caused by condom catheter. JAMA 1980; 244: 1.238 .

4. REHMAN M, RAB SM.: Penile necrosis caused by condom catheter. J Pak Med Assoc 1988; 38: 193-194.

5. JIMÉNEZ PENICK FJ, HERNÁNDEZ HORTELANO E, MORENO SIERRA J.: Incontinencia Urinaria y lesión medular traumática. Incontinencia Urinaria (Clínicas urológicas de la Complutense) 2000; 8: 349-374.

Dr. J.L. Palmero Martí

Servicio de Urología

Hospital Universitario La Fe

Avda. Campanar, 21

46009 Valencia

(Trabajo recibido el 13 junio de 2002) 